\title{
A Transformer Screens Discharge Analysis and Processing Method
}

\author{
Huang Yanhai, Zhang Bo, Guan Chunmei, Mu Jinglong, Zhao Chang, Wang Lei, \\ Cao Huiyan, Liu Ren, Li Feng, Zhang Xiaoshu, Xu Mei, Lan Chenghao, Jin Jing \\ Fushun Power Supply Company, Liaoning Electric Power Company Limited, State Grid, China \\ fushunpowersupply@163.com
}

Keywords: insulation; damp; block screens; dendritic discharge

\begin{abstract}
Combined with the actual set of concrete measures are for preventing the transformer screens dendritic discharge fault. Aiming at the $220 \mathrm{kV}$ main transformer screens dendritic discharge fault, introduce the basic process of screens dendritic discharge failure occurs, proof insulation damp and gas is the main external cause screens dendritic discharge fault.
\end{abstract}

\section{Introduction}

Transformer fault involves a wide range, most of the books、 papers just introduce find method of typical failure, such as short circuit fault, overheating fault, tap failure, insulation failure, discharge failure, core failure, etc.

Transformer screens dendritic discharge accident occurs mostly in three phase large transformers, unless the light gas relay, and timely inspection repair power outages; otherwise, when the heavy gas relay or differential protection action, the transformer will have serious deformation, sometimes the bolt fracture, along box, transformer oil leakage, consequence is very serious. In recent years, the technology of structure design of large transformer has made considerable progress, transformer screens dendritic discharge accident occurs rarely. But because each manufacturer's technical level and equipment conditions vary greatly, individual manufacturers or modified large transformer still screens dendritic discharge phenomenon occurs, discharge phenomenon, therefore, it is necessary to discuss the reasons and rectification measures of transformer screens dendritic discharge[1-3].

\section{The reasons of transformer screens discharge}

(1) The major external causes

Dry oil gap and cardboard dielectric strength of screen insulation structure is very high. However, oil immersed pressboard damp; the dielectric strength will be greatly reduced. Approach the tidal intrusion is generally safe airway, moisture absorber and transformer oil pump, conduit combining part. When the moisture through this way to enter the inside of the tank, resulting in transformer oil-paper insulation moisture oil paper insulation dielectric strength will be greatly reduced, resulting in screens dendritic discharge fault. The presence of gas in transformer oil is another cause of large transformers screens dendritic discharges. Because the gas will reduce the initial discharge large field strength, especially when high gas content in transformer, and moisture intrusion, if the screen board is attached to the surface moisture bubbles, or cardboard sandwich bubble, stratified and the existence of moisture hole, will be more easy to form the tree discharge[4].

In short, the transformer oil under strong electric field, produce gases, moisture in the oil(water content increase) is inevitable, in addition the North temperature difference, moisture and antipermeability, causing gas and water in oil. Insulating oil damp and gas is external reason of screens dendritic discharge occurred[5].

(2) Main internal

Insulation pads exist long corners a combination of insulation and oilpaper field strength depends on thickness of media pressure over the direction and uniformity of the media of the overall distribution if in the distribution of the insulating structure of high electric field region 
slightly lack, such as transformer winding pads and stays and other insulating structural parts exist sharp edges and corners, so that the wire electric field distortion. In addition to screen and winding medial exist prominent part bend transposition, winding rugged and cardboard bending deformation parts, narrow the local oil channel, so that both may cause local overheating, and can aggravate the electric field distortion, the field strength increases.

\section{The fault accident}

Two main transformers in a $220 \mathrm{kV}$ substation in parallel operation, load of $41000 \mathrm{~kW}$. $2 \#$ main transformer "body of light gas or pressure release valve action" light plate display, in section of No. two main transformer in appearance, the oil temperature and sound, but have not found abnormal, check the gas relay gas collecting chamber gas content is about the chamber set of $1 / 2$. The transformer winding deformation, the DC resistance, insulation resistance, DC leakage and dielectric loss angle test, results showed no abnormalities.

\section{The detection results}

Light gas signal appears, sampling the gas and oil service class oil chromatographic assay. The results are shown in table 1.

Table 1 The test data of oil chromatography after the accident

\begin{tabular}{|l|l|l|l|l|l|l|l|l|}
\hline \multicolumn{1}{|c|}{ CH4 } & \multicolumn{1}{c}{ C2H6 } & C2H4 & C2H2 & C1+C2 & \multicolumn{1}{c|}{ H2 } & \multicolumn{1}{c|}{ CO } & CO2 & The sampling sites \\
\hline 3017.61 & 68.17 & 675.52 & 1478.87 & 5240.17 & 23580 & 10950 & 5677 & Gas \\
\hline 163.94 & 53.39 & 251.01 & 298.30 & 766.70 & 596 & 1273 & 7817 & The device body oil 1 \\
\hline 155.87 & 52.79 & 254.25 & 307.23 & 770.13 & 544 & 1149 & 7547 & The device body oil 2 \\
\hline
\end{tabular}

Comparison of 2\# main transformer and out over the years in oil testing data, the test data are shown in Table 2.

Table 2 The oil chromatographic test data over years

\begin{tabular}{|c|c|c|c|c|c|c|c|c|}
\hline CH4 & C2H6 & C2H4 & C2H2 & C1+C2 & H2 & CO & CO2 & The sampling sites \\
\hline 18.01 & 12.46 & 42.52 & 0.06 & 73.05 & 43 & 572 & 1971 & The device body oil \\
\hline 17.27 & 5.16 & 38.43 & 0 & 60.86 & 35 & 710 & 2216 & The device body oil \\
\hline 19.41 & 12.88 & 47.67 & 0.22 & 80.18 & 28 & 679 & 2913 & The device body oil \\
\hline 30.40 & 9.12 & 50.21 & 0.22 & 89.95 & 47 & 1208 & 4787 & The device body oil \\
\hline 30.23 & 9.43 & 44.75 & 0.27 & 84.68 & 33 & 1044 & 3984 & The device body oil \\
\hline 35.15 & 11.13 & 47.50 & 0.16 & 93.94 & 35 & 904 & 5201 & The device body oil \\
\hline 41.75 & 12.46 & 50.03 & 0.22 & 104.46 & 34 & 1471 & 4988 & The device body oil \\
\hline 41.82 & 11.08 & 51.44 & 0.22 & 104.56 & 27 & 1010 & 7092 & The device body oil \\
\hline 3017.61 & 68.17 & 675.52 & 1478.87 & 5240.17 & 23580 & 10950 & 5677 & Gas \\
\hline 163.94 & 53.39 & 251.01 & 298.30 & 766.70 & 596 & 1273 & 7817 & The device body oil \\
\hline 155.87 & 52.79 & 254.25 & 307.23 & 770.13 & 544 & 1149 & 7547 & The device body oil \\
\hline
\end{tabular}

From the oil analysis data, hydrogen, acetylene, total hydrocarbon content exceed the standard seriously, from the characteristics of gas, acetylene content is high and constitute the main component of total hydrocarbon, and high content of hydrogen, can be judged for the discharge fault. Using three ratio methods: C2H2/ C2H4=307/254=1.2, Because the ratio of 1.2 between 1 and 3 , so the code is " $1 "$;

$\mathrm{CH} 4 / \mathrm{H} 2=155 / 544=0.3$, Because the ratio of 0.3 between 0.1 and 1 , so the code is " $0 "$;

$\mathrm{C} 2 \mathrm{H} 4 / \mathrm{C} 2 \mathrm{H} 6=254 / 53=4.8$, Because the ratio of 4.8 is greater than 3 , so the code is "2".

The three ratio is encoded as " $1,0,2$ ", the Department for the high energy discharge fault (i.e. 
arc discharge). From the gas analysis data, all kinds of fault gas concentration is far more than the concentration of oil gas, the fault gas in oil not dissolved, into gas, indicating that the transformer fault exists serious. From the contents of $\mathrm{CO}$ and $\mathrm{CO} 2$, the fault has been related to insulation board part.

\section{The disintegration test}

In the scene of the hanging core examination, lead no exception. Found traces of discharge on the A, B and white B-phase screens outermost layer of cardboard, from the longitudinal position of view, the high pressure outlet of the winding head end (central of high voltage winding) of the third downward, i.e. location winding Minister pad in contact with the screens, third winding has five burns, fourth paragraph windings have burn marks, which are shown in Figure 1 and Figure 2. Open the B-phase high-pressure screens, the innermost layer of cardboard found a large number of branches discharge burns. If not found, a short circuit accident will be lead to between the windings of the transformer.

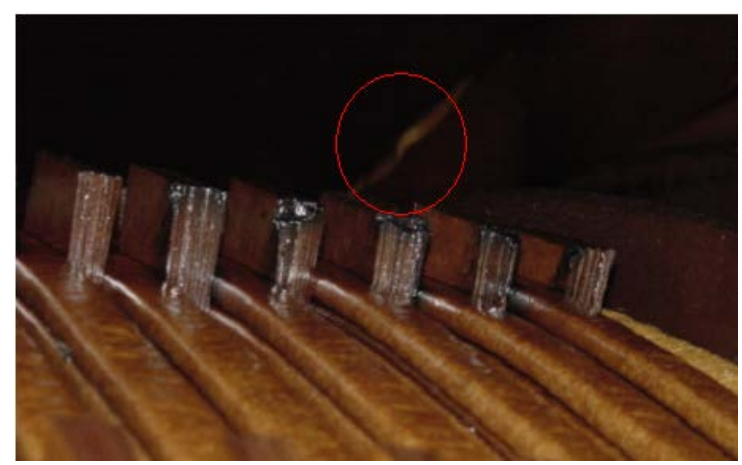

Figure 1 The discharge site from coil spacer to screen

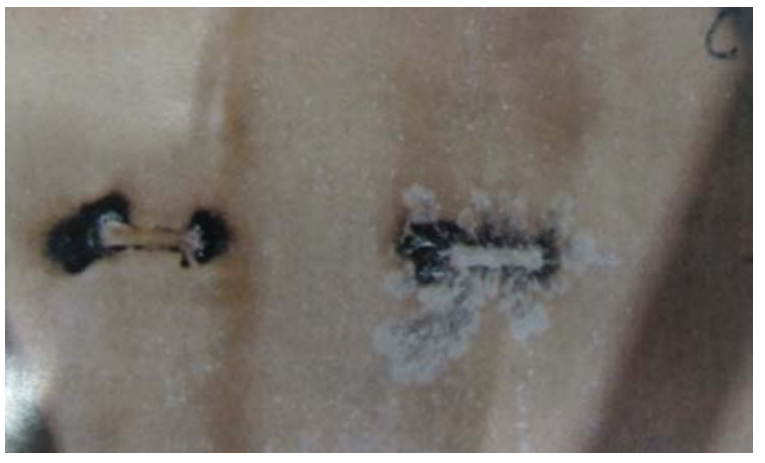

Figure 2 The discharge site on screen pad

\section{Fault handling}

In the middle of the first surface of the winding of the oil gap and add a layer of screens, the first original is divided into the oil clearance from $30 \mathrm{~mm}$ to $15 \mathrm{~mm}$, the middle phase insulating screens increase, thereby increasing the middle phase insulating level.

Pour into the R8 fillet reason: in the field of any member will with be potential, shape determines its field size. Because at the time of the design the long block is a rectangular, two sharp corners of the front end the first support to the screen board, in case of screens tight long cushion block linear of the front end part that is connected with the cardboard. This makes the screen board by high field effect in $\mathrm{M}$ and $\mathrm{N}$ points, but also to withstand screens tight mechanical action, discharge phenomenon first occurs in the $\mathrm{M}, \mathrm{N}$ points of long block front end, and connected with the screen board was burned and thus two outward expansion (in our actual observation also confirmed the two first appeared in discharge pattern), make the discharge burn marks develop along the board to the ground. Pour into R8 fillet can be limited to reduce electric field intensity. 


\section{Conclusion}

Through on-site installation and commissioning, vacuum oiling and the associated transformer oil chromatographic test, DC resistance test, ratio test, insulation resistance (absorption ratio) test, dielectric loss angle test winding deformation test, AC voltage test, and partial discharge test. After all tests are qualified projects, in at 15:00 on the 13th transmission. The author knows from the operation department, the transformer into operation is favorable safety operation.

\section{References}

[1] Dong Qiguo eds.. Power transformer fault and diagnosis. Chinese electric power press, 2000

[2] Chen Huagang. Electrical equipment preventive test questions and answers. Hydraulic and electric power press, 1998

[3] Chen Huagang. Electrical equipment preventive test method. Hydraulic and electric power press, 1999

[4] Cao Dunkui. Transformer oil chromatographic analysis and fault diagnosis. China Electric Power Press, 2011

[5] Wang Shige, Zhong Hongbi. Power transformer fault analysis and technical improvements. China Electric Power Press, 2004. 